\title{
Deficient maternal care resulting from immunological stress during pregnancy is associated with a sex-dependent enhancement of conditioned fear in the offspring
}

\author{
Severin Schwendener • Urs Meyer • Joram Feldon
}

Received: 17 July 2008 / Accepted: 27 October 2008 / Published online: 18 November 2008

(C) Springer Science + Business Media, LLC 2008

\begin{abstract}
Activation of maternal stress response systems during pregnancy has been associated with altered postpartum maternal care and subsequent abnormalities in the offspring's brain and behavioral development. It remains unknown, however, whether similar effects may be induced by exposure to immunological stress during pregnancy. The present study was designed to address this issue in a mouse model of prenatal immune activation by the viral mimic polyriboinosinic-polyribocytidilic acid (PolyI:C). Pregnant mice were exposed to PolyI:C-induced immune challenge or sham treatment, and offspring born to PolyI:C- and sham-treated dams were simultaneously cross-fostered to surrogate rearing mothers, which had either experienced inflammatory or vehicle treatment during pregnancy. We evaluated the effects of the maternal immunological manipulation on postpartum maternal behavior, and we assessed the prenatal and postnatal maternal influences on anxietyand fear-related behavior in the offspring at the periadolescent and adult stage of development. We found that PolyI:C treatment during pregnancy led to changes in postpartum maternal behavior in the form of reduced pup licking/grooming and increased nest building activity. Furthermore, the adoption of neonates by surrogate rearing mothers, which had experienced PolyI:C-induced immunological stress during pregnancy, led to enhanced conditioned fear in the peri-adolescent and adult offspring, an effect that was exclusively seen in female but not male
\end{abstract}

S. Schwendener and U. Meyer contributed equally to this work.

S. Schwendener $\cdot$ U. Meyer $\cdot$ J. Feldon $(\bowtie)$

Laboratory of Behavioural Neurobiology, ETH Zurich,

Schorenstrasse 16,

8603 Schwerzenbach, Switzerland

e-mail: feldon@behav.biol.ethz.ch subjects. Unconditioned (innate) anxiety-related behavior as assessed in the elevated plus maze and open field explorations tests were not affected by the prenatal and postnatal manipulations. Our results thus highlight that being raised by gestationally immune-challenged surrogate mothers increases the vulnerability for specific forms of fear-related behavioral pathology in later life, and that this association may be mediated by deficits in postpartum maternal care. This may have important implications for the identification and characterization of early-life risk factors involved in the developmental etiology of fear-related neuropsychiatric disorders.

Keywords Adoption · Anxiety · Fear - Infection · Maternal behavior Pregnancy

\section{Introduction}

Maternal infection during pregnancy can lead to direct physiological changes in the fetal environment, which may negatively influence normal brain and behavioral development in the offspring [56, 57]. Indeed, prenatal exposure to infection has been identified as an environmental risk factor for the development of severe brain disorders in later life, including schizophrenia [6, 5, 52], autism [2, 11], and cerebral palsy [17]. Experimental investigations in rodent models of prenatal immune challenge have also provided considerable support for the biological plausibility and causality in the link between in utero exposure to infection and higher risk of postnatal brain dysfunctions (for recent reviews see [37, 43, 46, 66]. Infection-induced disruption of fetal brain development may thus predispose the organism for long-lasting structural and functional brain abnormalities, leading to the emer- 
gence of neuropathology and psychopathology in postnatal life $[3,22,38,51]$.

In addition to the prenatal maternal effects, immune activation during pregnancy may also induce significant changes in postpartum maternal factors. It is known that immunological stimulation is linked to the activation of several stress response systems, including the hypothalamicpituitary-adrenal (HPA) axis [23, 69]; and that maternal physiological and/or psychological stress during pregnancy can alter postpartum maternal behavior [34, 50, 63]. In both humans and rodents, disruption of the intricate motherinfant relationship may be a critical postnatal factor for the induction of adult psychopathological behavior [9, 10, 12, 49 , 53]. Hence, changes in postnatal maternal factors resulting from immunological stress during pregnancy may subject the neonate to an adverse rearing environment and thereby confer additional risk for abnormal brain and behavioral development in the offspring.

We have recently found initial experimental evidence for this possibility by showing that the adoption of control neonates by surrogate rearing mothers, which had been exposed to immune activation during pregnancy, is sufficient to induce impaired cognitive development and enhanced sensitivity to psychostimulant drugs in the fostered offspring [40, 42]. However, the critical postnatal factors underlying this association have not been identified yet. Specifically, it remains unknown whether gestational immune challenge is associated with alterations in postpartum maternal behavior, and whether this may be a critical mediating mechanism for altered brain and behavioral development in the fostered offspring.

Therefore, the present study was designed to explore these issues in a mouse model of prenatal immune activation by the synthetic analogue of double-stranded RNA, polyriboinosinic-polyribocytidilic acid (PolyI:C). PolyI:C mimics the acute phase response to viral infection, which is accompanied by the presence of high levels of proinflammatory cytokines and other mediators of inflammation [16, 21, 39]. PolyI:C treatment also activates the HPA axis via stimulation of corticotrophin-releasing factor secretion [44]. Moreover, prenatal PolyI:C exposure is known to induce to a variety of postnatal structural and functional brain abnormalities in both rats and mice [36, 38, 39, 40-42, 61, 64, 72, 74]. Maternal administration of PolyI:C during pregnancy is thus a valuable experimental tool to study the effects of fetal brain inflammation on subsequent brain development, and to explore the influence of gestational immune challenge on postpartum maternal behavior.

In order to be able to dissect prenatal and postnatal maternal factors, we combined the prenatal PolyI:Cinduced immunological manipulation with a postnatal cross-fostering design. Pregnant mice in late gestation were exposed to PolyI:C-induced immune activation or sham treatment, and offspring born to PolyI:C- and sham-treated dams were simultaneously cross-fostered to surrogate rearing mothers, which had either experienced inflammatory or vehicle treatment during pregnancy. On the one hand, we assessed postpartum maternal behavior after completion of the cross-fostering procedure; and on the other hand, we studied the effects of the prenatal and postnatal manipulations on anxiety and fear-related behavior in the fostered offspring when they reached peri-adolescence and adulthood. Unconditioned (innate) anxiety-like behavior was assessed using the elevated plus maze and open field exploration tests [4], and conditioned fear was studied in the paradigm of classical (Pavlovian) fear conditioning [19]. These paradigms were selected primarily because they are known to be sensitive assays for the detection of abnormal anxiety- and fear-related behaviors emerging in animals reared under deficient maternal care (see e.g., [8, 35, 67]).

\section{Materials and methods}

\section{Animals}

Female and male C57BL6/J breeders were obtained from our in-house specific pathogen-free (SPF) colony at the age of 10-14 weeks. Littermates of the same sex were kept in groups of three to five mice. Breeding began after 2 weeks of acclimatization to the new animal holding room, which was a temperature- and humidity-controlled $\left(21 \pm 1^{\circ} \mathrm{C}, 55 \pm\right.$ $5 \%$ ) holding facility under a reversed light-dark cycle (lights off 07.00-19.00). All animals had ad libitum access to food (Kliba 3430, Klibamühlen, Kaiseraugst, Switzerland) and water. The breeding procedure and the verification of pregnancy have been fully described elsewhere [36]. All procedures described in the present study had been approved previously by the Zurich Cantonal Veterinary Office and are in agreement with the Principles of Laboratory Animal Care (National Institutes of Health publication number 86-23, revised 1985).

\section{Maternal immune treatment during pregnancy}

Pregnant dams on gestation day 17 (GD17) received a single injection of PolyI:C (potassium salt; Sigma-Aldrich, Buchs, St. Gallen, Switzerland) or vehicle. PolyI:C (5 mg/kg; calculated based on the pure form PolyI:C) was dissolved in isotonic $0.9 \% \mathrm{NaCl}$ solution to yield a final concentration of $1 \mathrm{mg} / \mathrm{ml}$. The dose of PolyI:C is identical to the one used in our previous studies conducted in C57BL/6 mice (e.g., $[36,39,42])$. The gestational window (GD17) was selected based on our previous observations (e.g., [39, 41]) and corresponds roughly to the middle-to-late second trimester 
of human pregnancy with respect to developmental biology and percentage of gestation from mice to humans [13, 24]. All solutions were administered via the intravenous route (i.v.) at the tail vein under mild physical constraint as described before [36]. The injection volume was $5 \mathrm{ml} / \mathrm{kg}$. All animals were returned to their home cages immediately after the injection procedures and left undisturbed until the postnatal cross-fostering procedure (see below).

\section{Postnatal cross-fostering}

On the day of birth (postnatal day [PND] 1), offspring born to PolyI:C- and vehicle-treated dams were culled to litters of six to eight animals each (with three to four animals per sex) and cross-fostered to surrogate rearing mothers. Male and female pups were removed from the original mother and gently placed in a cage containing sawdust bedding for a maximum of $10 \mathrm{~min}$. Half of a given litter was then placed with a PolyI:C-treated surrogate rearing mother and the other half with a vehicle-treated rearing mother. Each surrogate mother thus simultaneously fostered pups originating from both prenatal treatment conditions, but not its own offspring. PolyI:C and vehicle offspring were marked on the left and right hind paw, respectively. A total of 16 litters (eight PolyI:C and eight vehicle) were cross-fostered to 16 rearing mothers, half of which had been subjected to PolyI:C exposure during pregnancy and the other half to vehicle treatment only.

The cross-fostering resulted in four experimental treatment groups (see Fig. 1): (1) offspring subjected to prenatal
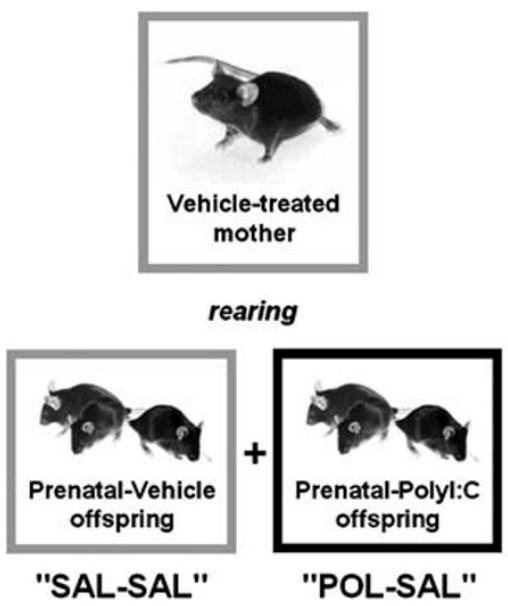

Fig. 1 Experimental design for the postnatal cross-fostering procedure. Pregnant mice on gestation day 17 were exposed to PolyI:C (POL; $5 \mathrm{mg} / \mathrm{kg}$, i.v.) or saline ( $S A L)$ solution. On the day of birth, offspring born to PolyI:C- and saline-treated dams were simultaneously crossfostered to surrogate rearing mothers, which had either experienced inflammatory (PolyI:C) or sham (saline) treatment during pregnancy. This cross-fostering procedure resulted in four experimental treatment vehicle exposure and raised by a vehicle-treated surrogate mother (SAL-SAL), (2) offspring subjected to prenatal vehicle exposure and raised by a PolyI:C-treated surrogate mother (SAL-POL), (3) offspring subjected to prenatal PolyI:C exposure and raised by a vehicle-treated surrogate mother (POL-SAL), and (4) offspring subjected to prenatal PolyI:C exposure and raised by a PolyI:C-treated surrogate mother (POL-POL).

The animals were kept in a temperature- and humiditycontrolled $\left(21 \pm 1^{\circ} \mathrm{C}, 55 \pm 5 \%\right)$ holding facility under a reversed light-dark cycle (lights off 07.00-19.00), and they had ad libitum access to food and water as described above. The animal cages were cleaned once a week, beginning from the postnatal cross-fostering procedure on PND 1. This was conducted always by the same person.

Assessment of maternal behavior

The apparatus consisted of 16 transparent polycarbonate cages $(45 \times 24 \times 19 \mathrm{~cm}$ high) filled with sawdust. On the grid top of each cage, a digital video camera (CCD B/W $500 \times$ 500 SX200B-2; Profitronic, Aarau, Switzerland) was mounted for the purpose of video recordings. The output of the camera was fed to a multiplexer (YSQ-430, Sony, Japan) before being transmitted to a PC running the Windows XP operating system and the WINTV-2000 program for data acquisition. The multiplexer was programmed in such a way that it switched from one cage to the next every $20 \mathrm{~s}$. Recording took place every $3 \mathrm{~h}$, with one recording session lasting $20 \mathrm{~min}$. Hence, there were a total of eight 20-min

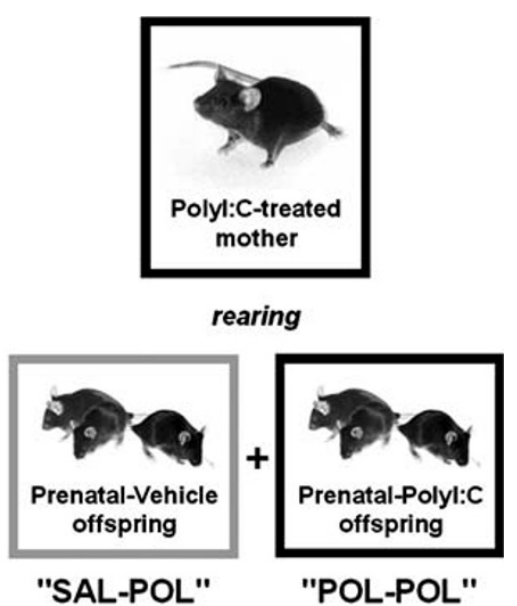

groups: (1) offspring subjected to prenatal saline exposure and raised by a saline-treated surrogate mother (SAL-SAL), (2) offspring subjected to prenatal saline exposure and raised by a PolyI:C-treated surrogate mother (SAL-POL), (3) offspring subjected to prenatal PolyI:C exposure and raised by a saline-treated surrogate mother (POL-SAL), and (4) offspring subjected to prenatal PolyI:C exposure and raised by a PolyI:C-treated surrogate mother (POL-POL) 
recording sessions per $24 \mathrm{~h}$ (starting at 02.30, 05.30, 08.30, $11.30,14.30,17.30,20.30$, and 23.30), with each session allowing three to four 20 -s observations per cage.

The recording of maternal behavior started after completion of the cross-fostering procedure on PND 1 (see above) and lasted for one week (i.e., until PND 7). We focused on this period because maternal observations over the first week postpartum are expected to be most critical for the reliable assessment of possible differences in maternal behavior (see [9]).

Maternal behavior was subdivided into the following categories, adapted from the definitions by [30] and [59].

Licking/grooming The mother is licking and/or grooming the pups, clearly recognizable by the movement of her head and/or paws.

Self-licking/-grooming This refers to self-directed licking and/or grooming behavior when the mother is in the nest; self-directed licking and/or grooming behavior occurring outside the nest would be scored as "off-nest" (see below).

Eating/drinking The mother is eating and drinking from food magazine and water bottles, respectively.

Nursing This refers to feeding the pups; this comprises all categories of nursing, including kyphosis (nursing the pups in a dorsal upright position, with rigid fore- and/or hindlimbs) and prone nursing (nursing the pups while lying flat on the top of the pups, with little or no limb support).

Nest-building The mother is moving sawdust around the nest or is preparing a new nest; in addition, moving pups from one nest to another is included in this category.

Off nest This is scored when the mother is somewhere in the cage without any contact to the pups; this category does not include the categories of "self-licking/-grooming", "eating/drinking" or "nest-building".

Undefined This is scored when it is not possible to clearly assign maternal behavior to one of the above categories.

For the purpose of analysis, each individual maternal behavior was expressed as a proportion (in percent) of the total maternal behavioral scores by using the formula: [maternal behavioral scores in one specific category/ maternal behavioral scores in all categories $] \times 100$.

Behavioral testing of offspring

All offspring were weaned at PND 24. The pups were weighed and littermates of the same sex were caged separately, thereby separating the animals according to the four treatment conditions. All animals were maintained under ad libitum food (Kliba, 3430, Klibamühlen, Kaiseraugst, Switzerland) and water diet, and kept in a temperature and humidity-controlled $\left(21 \pm 1{ }^{\circ} \mathrm{C}, 55 \pm 5 \%\right)$ animal vivarium under a 12:12 h reversed light-dark cycle (lights off 07.0019.00) as described before.

Experiments 1-3 were conducted in peri-adolescence, that is, when the offspring reached PND 35-45. Each of the four treatment groups consisted of subjects derived from multiple independent litters formed by the cross-fostering procedure in order to minimize potential confounds resulting from litter effects [71]. Experiments 4-6 were conducted in a new cohort of experimentally naïve animals when they reached adult age (i.e. between PND 85-95). Again, the experiments included testing of offspring derived from multiple litters. All experiments included testing of male and female animals. The number of subjects employed in each of the behavioral tests is listed in Table 1 .

Peri-adolescence was defined as the period between PND 28 and 45, and adulthood was defined as the period of PND 65 onward, according to the gradual attainment of sexual maturity and age-specific behavioral discontinuities from younger to older animals [65]. These two developmental stages are largely comparable between mice and rats, and correspond to a period between $11-16$ and 20 years onward, respectively, in humans [55, 62, 65].

\section{Elevated plus maze test}

The elevated plus maze was made of opaque acryl glass, and elevated at a height of $70 \mathrm{~cm}$ above floor level. It consisted of four equally spaced arms radiating out from a central square measuring $5 \times 5 \mathrm{~cm}$. Each arm was $30 \mathrm{~cm}$ long. Two opposing arms were enclosed by $15 \mathrm{~cm}$ high opaque walls from all sides except the side adjoining the central square. The other two arms were exposed and the outer rim of the open arms was guarded by a perimeter border of $1 \mathrm{~mm}$. The floor of the entire maze was covered by a grey plastic inlay that could be easily removed and cleansed with water between trials. The maze was located in a dimly lit experimental room. The light level in the open arms of the maze was balanced at $30 \mathrm{~lx}$. A digital camera was mounted above the maze and images were transmitted at a rate of $5 \mathrm{~Hz}$ to a personal computer running the Ethovision (Noldus IT, Wageningen, The Netherlands) software allowing the tracking of the subject's position.

To begin a trial, the mouse was placed in the center of the maze facing one of the open arms. It was allowed to move freely undisturbed for 5 min before being returned to the home cage. The dependent measures were (a) total distance traversed in the entire maze, (b) distance moved in the open arms, (c) frequency of open arm entries, and (d) time spent in the open arms. Frequency of arm entries and 
Table 1 Summary of the number of subjects employed in the behavioral tests

\begin{tabular}{|c|c|c|c|c|c|}
\hline \multirow[t]{2}{*}{ Experiments } & \multicolumn{4}{|c|}{ Experimental groups (prenatal treatment-postnatal rearing) } & \multirow[t]{2}{*}{ Age (PND) } \\
\hline & SAL-SAL & SAL-POL & POL-SAL & POL-POL & \\
\hline 1. Elevated plus maze & $5 \hat{\jmath}, 5$ q & $6 \hat{\jmath}, 4 q$ & $5 \hat{\jmath}, 5 q$ & $5 \hat{\jmath}, 5 q$ & 35 \\
\hline 2. Open field & $5 \hat{\jmath}, 5$ 우 & $6 \hat{\jmath}, 4 q$ & $5 \hat{\jmath}, 5 q$ & $5 \hat{\jmath}, 5$ 우 & 38 \\
\hline 3. Fear conditioning & $5 \hat{\jmath}, 5 q$ & $6 \hat{\jmath}, 4 q$ & $5 \hat{\jmath}, 5 q$ & $5 \hat{\jmath}, 5$ ㅇ & $42-44$ \\
\hline 4. Elevated plus maze & $5 \hat{\jmath}, 5 q$ & $6 \hat{\jmath}, 4 q$ & $4 \hat{\jmath}, 6$ 守 & $5 \hat{\jmath}, 5$ 우 & 85 \\
\hline 5. Open field & $5 \hat{\jmath}, 5$ q & $6 \hat{0}, 4$ + & $4 \hat{\jmath}, 6$ 우 & $5 \hat{0}, 5$ ㅇ & 88 \\
\hline 6. Fear conditioning & $5 \hat{\jmath}, 5 q$ & $6 \hat{0}, 4$ & $4 \hat{\jmath}, 6$ 우 & $5 \hat{\jmath}, 5$ ㅇ & $91-93$ \\
\hline
\end{tabular}

Neonates of saline (SAL)- and PolyI:C (POL)-treated dams were cross-fostered to surrogate-rearing mothers, which had been exposed to either saline or PolyI:C during pregnancy. This yielded four experimental groups according to a two (prenatal treatment) $\times$ two (postnatal rearing) design. Experiments 1-3 assessed anxiety- and fear-related behavior when the fostered offspring reached peri-adolescence, i.e., between postnatal days (PNDs) 35 and 44. The same subjects were used for repeated testing at the indicated order. Experiments 4-6 assessed anxiety- and fearrelated behavior in a new cohort of fostered offspring when they reached adulthood, i.e., at PND 85-93. Again, the same subjects were used for repeated testing at the indicated order. The offspring were derived from 16 mothers, half of which had been subjected to PolyI:C exposure during pregnancy and the other half to vehicle treatment only. All experiments included testing of male and female animals

time spent in the open arms were expressed as percent scores over the total number of all (open and enclosed) arm entries, and total time spent in all arms, respectively.

\section{Open field test}

The open field exploration tests were conducted in four identical square arenas $(40 \times 40 \times 35 \mathrm{~cm}$ high $)$ made of wood and painted white. They were located in a testing room under diffused lighting (approximately $35 \mathrm{~lx}$ as measured in the center of the arenas). A digital camera was mounted directly above the four arenas. Images were captured at a rate of $5 \mathrm{~Hz}$ and transmitted to a PC running the Ethovision (Noldus, The Netherlands) tracking system.

The animals were gently placed in the center of the arena and allowed to explore for $1 \mathrm{~h}$. For the purpose of data collection, the arena was conceptually partitioned into two zones: a center zone (measuring $13.5 \times 13.5 \mathrm{~cm}$ ) in the middle of the arena and a peripheral zone occupying the remaining area. The dependent measures were (a) distance moved in the entire arena, (b) number of entries into the center zone, (c) time spent in the center zone, and (d) distance travelled in the center zone. These were expressed and analyzed as a function of 10-min bins.

\section{Classical fear conditioning}

The apparatus comprised two sets of four test chambers to provide two distinct contexts. The first set of chambers (context A) comprised four Coulbourn Instruments (P.A., USA) operant chambers (Model E10-10) each installed in a ventilated, sound-insulated chest. The chamber of context A measured $30 \times 25 \times 29$ (high) $\mathrm{cm}$, but the animal was confined to an area of $17.5 \times 13 \mathrm{~cm}$ in the center by a transparent Plexiglas enclosure. Illumination inside the chamber was provided by a house light $(2.8 \mathrm{~W})$ positioned on the panel wall, $21 \mathrm{~cm}$ above the grid floor. The grid floor was made of stainless steel rods (4 $\mathrm{mm}$ in diameter) spaced at regular intervals of $10 \mathrm{~mm}$ center to center, and through which scrambled electric foot shock at $1 \mathrm{~s}$ duration and $0.3 \mathrm{~mA}$ intensity (the unconditioned stimulus, US), generated by a shock scrambler (Model E13-14), could be delivered. The second set of chambers (context B) comprised four cylindrical (19 $\mathrm{cm}$ in diameter) enclosures made of clear Plexiglas and painted in light grey, rested on a metal mesh floor; each enclosure was located in a sound-insulated, wooden cabinet. It was illuminated by an infrared light source instead of visible light. All eight chambers contained a sonalert unit (Model H12-02M-2.9), which could deliver a $2.9 \mathrm{kHz}$ tone measuring approximately $86 \mathrm{~dB}_{\mathrm{A}}$. This provided the conditioned stimulus (CS). In addition, a miniature digital camera was mounted $30 \mathrm{~cm}$ directly above the center of the area of interest. The output of the camera was fed to a multiplexer (YSQ-430, Sony, Japan) before being transmitted to a PC running the NIH Image software (version 1.61) for real-time analysis. The algorithm of the freezing response detection procedure has been validated and fully described before [58]. Briefly, successive digitized images $(192 \times 144=27,648$ pixels, at 8 -bit grey scale $)$ obtained at a rate of $1 \mathrm{~Hz}$ were compared. The number of pixels differing between adjacent frames was then computed. If this was less than $0.05 \%$ of the total number of pixels in a frame, the animal was considered to be freezing in that 1-s interval.

On day 1, all animals were given three separate CS-US (tone-shock) pairings, presented at 3-min intervals, in context A. In each pairing, the 1-s shock US immediately followed the 30-s tone CS. On the day of conditioning, the amount of freezing during the three occasions of tone presentation provided a measure for the evaluation of the acquisition of conditioning. 
On day 2, the animals were returned to context A. They were placed in the test chamber for a period of $6 \mathrm{~min}$. This served as a test of context freezing. The expression of context freezing was indexed as percent time freezing across the 6 min period.

On day 3, CS-freezing to the tone stimulus was assessed in context $\mathrm{B}$. The tone stimulus was administered $3 \mathrm{~min}$ after the animals were placed into the test chamber. The tone remained on for a period of $6 \mathrm{~min}$, to parallel the test period of context freezing.

\section{Statistical analysis}

All data were analyzed using parametric analysis of variance (ANOVA), followed by Fisher's Least Significant Difference (LSD) post hoc comparisons or restricted ANOVAs whenever appropriate. Statistical significance was set at $p<0.05$. All statistical analyses were conducted using the statistical software StatView (version 5.0) implemented on a personal computer running the Windows XP operating system.

\section{Results}

Effects of maternal immune activation during pregnancy on post-partum maternal behavior

The percent scores of each individual maternal behavior (i.e., [maternal behavioral scores in one specific category/ maternal behavioral scores in all categories] $\times 100$ ) were analyzed using $2 \times 8 \times 7$ (prenatal treatment $\times$ daytime $\times$ days) split-plot ANOVAs.

Prenatal PolyI:C-induced immune challenge led to a significant overall reduction in licking/grooming of pups compared to prenatal vehicle treatment, as supported by the significant main effect of prenatal treatment $\left(F_{1,14}=4.9, p<\right.$ 0.05). As depicted in Fig. 2, this effect of the prenatal immunological manipulation was most pronounced in the first three days postpartum. Licking/grooming behavior also significantly varied as a function of daytime, with maximal and minimal levels occurring between 20.30-20.50 and $02.30-02.50$, respectively. This fluctuation of licking/ grooming behavior was not significantly influenced by the prenatal immunological manipulation. These impressions were supported by the presence of a significant main effect of daytime $\left(F_{7,98}=2.5, p<0.05\right)$, with no other main effects or interactions attaining statistical significance in the ANOVA of licking/grooming.

In contrast to pup-directed grooming/licking behavior, selflicking/-grooming was not significantly affected by prenatal exposure to immune activation (Fig. 2). For both experimental groups, self-licking/-grooming was highest at PND 1 and subsided afterwards to reach minimal levels at PND 7. This led to a significant main effect of days $\left(F_{6,84}=3.7, p<0.01\right)$. No other main effects or interactions reached statistical significance in the ANOVA of self-licking/-grooming.

In comparison with controls, however, maternal immune activation during pregnancy significantly increased postpartum nest building behavior, an effect that was most pronounced at PND 1 and 2 (Fig. 2). Statistical support for this interpretation was yielded by the significant main effect of prenatal treatment $\left(F_{1,14}=4.5, p<0.05\right)$ and its interaction with days $\left(F_{6,84}=3.1, p<0.05\right)$. Additional $2 \times 8$ (prenatal treatment $\times$ daytime) ANOVAs restricted to each day were then performed. These analyses confirmed that maternal PolyI:C exposure significantly increased nest building behavior at PND $1\left(F_{1,14}=5.7, p<0.05\right)$ and PND $2\left(F_{1,14}=\right.$ $4.9, p<0.05)$ in comparison with maternal vehicle treatment. In general, nest building behavior varied across daytime: Engagement in nest building was minimal at 02.30 , but then gradually increased to reach maximal levels at 20.30. This daytime-dependent variation in nest building behavior was not significantly influenced by the prenatal immunological manipulation.

Maternal PolyI:C-induced immune activation during pregnancy did not exert any significant influence on nursing of pups, eating/drinking, or off-nest behavior (Fig. 2). Pup nursing was highest at PND 2 and subsided afterwards, whilst eating/drinking and off-nest scores reached maximal levels between PND 5 and PND 7 (Fig. 2). This pattern of results led to significant main effects of days in the ANOVAs of these measures (nursing: $F_{6,84}=8.3, p<0.001$; eating/drinking: $F_{6,84}=3.6, p<0.01$; off-nest: $F_{6,84}=5.3, p<$ 0.001 ). All three measures also varied as a function of daytime, as indicated by the main effect of daytime (nursing: $F_{7,98}=2.6, p<0.05$; eating/drinking: $F_{7,98}=4.6, p<0.001$; off-nest: $\left.F_{7,98}=3.5, p<0.01\right)$. For both eating/drinking and off-nest activities, maximal and minimal eating/drinking scores were obtained during recording sessions performed in the first half of the dark phase (i.e., at $08.30-08.50$ and 11.30-11.50) and in the middle of the light phase (i.e., at 23.30-23.50 and 02.30-02.50) of the light-dark cycle, respectively. On the other hand, nursing activity was highest in the middle of the light phase of the light-dark cycle (i.e., at 23.30-23.50 and 02.30-02.50), and it was lowest approximately in the middle of the dark phase (i.e., at $11.30-11.50)$.

Effects of the prenatal and postnatal manipulations on the offspring's body weight

\section{- Birth}

The body weight of pups in each litter was averaged per sex and analyzed using a $2 \times 2$ (prenatal treatment $\times$ sex $)$ 
LICKING / GROOMING
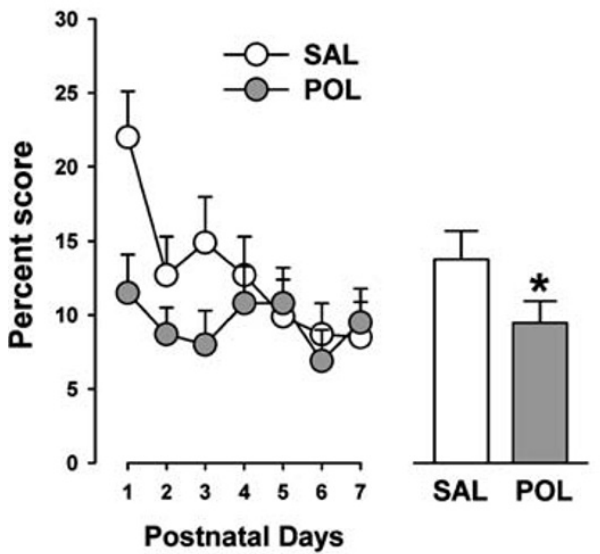

NURSING

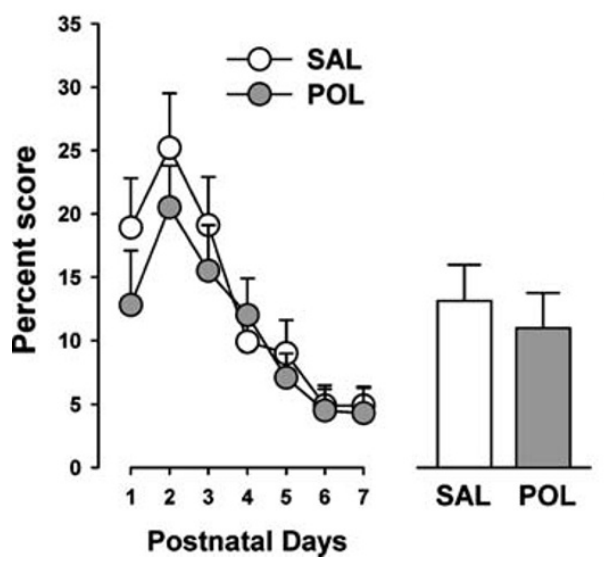

Fig. 2 Effects of maternal immune challenge in late gestation on postpartum maternal behavior. Pregnant mice on gestation day 17 were subjected to the viral mimic PolyI:C $(P O L)$ or vehicle (saline, $S A L$ ) treatment, and maternal behavior was assessed from postnatal days 1-7. Each individual maternal behavior was expressed as a

randomized block ANOVA. Maternal PolyI:C-induced immune challenge during pregnancy did not significantly affect the offspring's body weight on the day of birth (PND 1). Furthermore, no significant differences were detected between male and female pups at PND 1. The overall mean body weight $( \pm$ SEM) of pups at PND 1 was $1.24 \pm 0.02 \mathrm{~g}$.

\section{- Weaning}

The body weight of offspring in each litter formed in the cross-fostering procedure was averaged per sex and analyzed using a $2 \times 2 \times 2$ (prenatal treatment $\times$ postnatal rearing $\times$ sex) randomized block ANOVA. On the day of weaning (PND 24), the body weight of female subjects was generally lower compared to male animals, as indicated by the presence of a significant main effect of $\operatorname{sex}\left(F_{1,43}=4.8\right.$, $p<0.05)$. The overall mean body weight $( \pm \mathrm{SEM})$ of female and male subjects at PND 24 was $10.10 \pm 0.27$ and $11.79 \pm$
NEST BUILDING

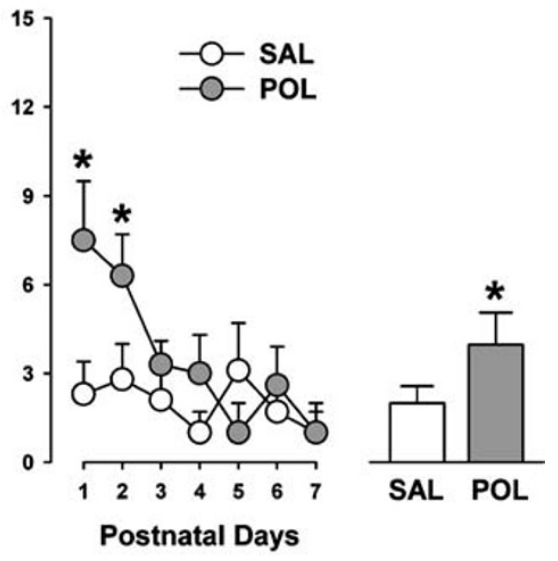

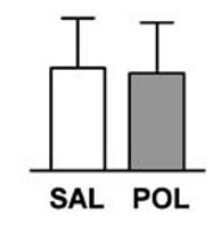
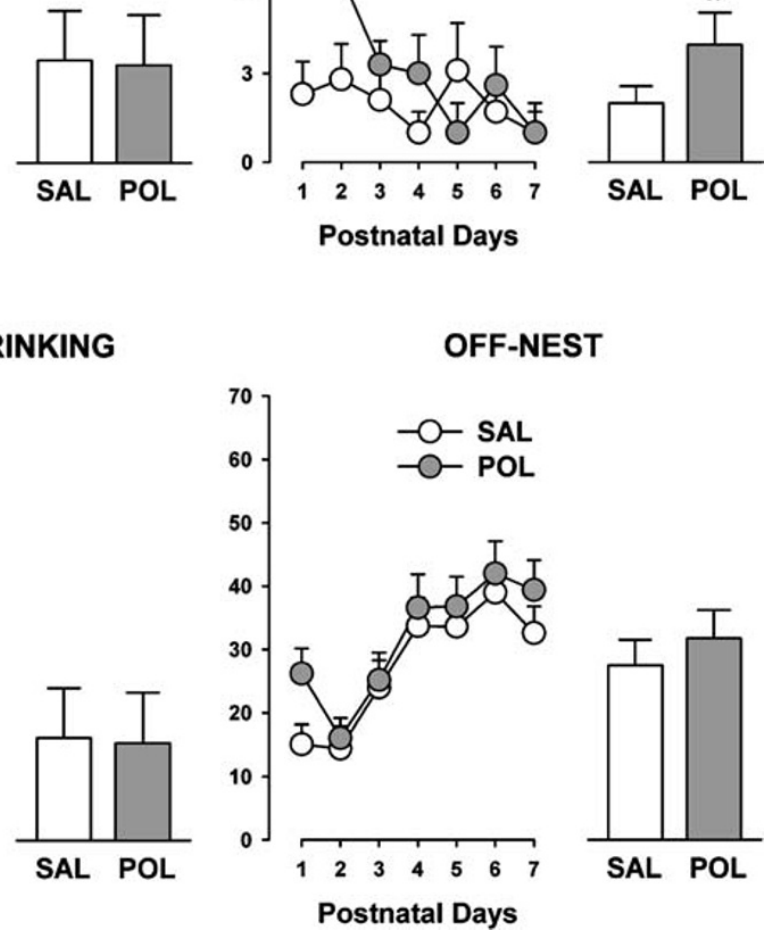

proportion (in percent) of the total maternal behavioral scores, and is depicted as a function of postnatal days (line plots) as well as average over the 7-days observation period (bar plots). For both experimental conditions, $N=8$. Symbol $(*)$ refers to a statistical significance of $p<$ 0.05 . All values are means \pm SEM

$0.23 \mathrm{~g}$, respectively. In addition, a small but significant reduction in body weight was noted in offspring of mothers exposed to PolyI:C treatment during pregnancy in comparison with offspring born to vehicle-treated mothers. This effect was independent of the postnatal rearing condition and sex. These impressions were supported by the main effect of prenatal treatment attaining statistical significance $\left(F_{1,43}=\right.$ $4.2, p<0.05)$. The overall mean body weight $( \pm \mathrm{SEM})$ of prenatally PolyI:C- and vehicle-exposed offspring was $10.14 \pm 0.32$ and $11.18 \pm 0.20 \mathrm{~g}$, respectively. No other main effects of interactions reached statistical significance.

\section{- Adulthood}

The body weight of offspring in each litter formed in the cross-fostering procedure was averaged per sex and analyzed using a $2 \times 2 \times 2($ prenatal treatment $\times$ postnatal rearing $\times$ sex $)$ randomized block ANOVA as described before. At adult age 
(PND 80), the body weight of female subjects was significantly lower compared to male animals, regardless of the prenatal treatment histories and postnatal rearing condition. Indeed, the prenatal and postnatal maternal manipulations did not significantly affect the offspring's body weight in adulthood. ANOVA only revealed a significant main effect of $\operatorname{sex}\left(F_{1,32}=73.8, p<0.001\right)$. The overall mean body weight $( \pm$ SEM $)$ of female and male subjects at PND 80 was $18.91 \pm 0.37$ and $23.78 \pm 0.39 \mathrm{~g}$, respectively.

Effects of the prenatal and postnatal manipulations on locomotor activity and unconditioned anxiety-related behaviors in peri-adolescence

\section{Elevated plus maze test}

First, we assessed the effects of the prenatal (PolyI:C exposure) and postnatal (cross-fostering) manipulations on the offspring's performance in the elevated plus maze test when they reached the peri-adolescent stage of development. Neither the prenatal nor the postnatal manipulation exerted a significant influence on anxiety-related behaviors in periadolescent subjects as indexed by the percent time spent in the open arms, percent open arm entries, and distance moved in the open arms. No significant main effects or interactions were obtained in the $2 \times 2 \times 2$ (prenatal treatment $\times$ postnatal rearing $\times$ sex) ANOVA of these measures. General locomotor activity was also largely comparable between the four experimental groups, as well as between male and female subjects. Locomotor activity was indexed by the total distance moved and analyzed by identical ANOVA, which yielded no significant main effects or interactions. The means \pm SEM of total distance moved, distance moved in the open arms, percent time spent in the open arms, percent open arm entries for each experimental group are provided in Table 2.

\section{Open field exploration test}

Consistent with the results obtained in the elevated plus maze test, exploratory behaviors were not significantly affected by the prenatal immunological manipulation or the postnatal rearing condition in the open field test at periadolescent age. The $2 \times 2 \times 2 \times 6$ (prenatal treatment $\times$ postnatal rearing $\times$ sex $\times 10$-min bins) ANOVA of center zone entries, time spent in the center zone, or distance moved in the center zone did not reveal any significant main effects or interactions involving the between-subjects factors of prenatal treatment, postnatal rearing or sex. In all experimental groups, the amount of time spent in the center zone, center zone entries, and distance moved in the center zone decreased across the six 10-min bins, indicating habituation effects. Statistical support for this interpretation was yielded by the ANOVAs of these measures, which revealed significant main effects of 10 -min bins (center zone entries: $F_{5,160}=34.8, p<0.001$; center zone time: $F_{5,160}=4.2, p<0.01$; distance moved in the center zone time: $\left.F_{5,160}=27.57, p<0.001\right)$. There was also a clear habituation effect with a reduction of locomotor activity over time, and this was evident in all experimental groups regardless of sex. This was supported by the ANOVA of distance traveled, which revealed a significant main effect of 10 -min bins $\left(F_{5,160}=144.5, p<0.001\right)$. Female subjects displayed a general increase in locomotor activity compared to male subjects, leading to the presence of significant main effect of $\operatorname{sex}\left(F_{1,32}=8.7, p<0.01\right)$ in the ANOVA of total distance traveled. No main effects or interactions involving

Table 2 Summary of the effects of the prenatal and postnatal maternal manipulations on the offspring's performance in the elevated plus maze and open field exploration tests in peri-adolescence

\begin{tabular}{lccc}
\hline Experiment & Experimental groups (prenatal treatment-postnatal rearing) & \\
\cline { 2 - 4 } & SAL-SAL & SAL-POL & POL-SAL \\
\hline Elevated plus maze & & & \\
Total distance moved (m) & $14.10 \pm 2.31$ & $15.84 \pm 2.04$ & $18.82 \pm 3.83$ \\
Distance moved (m) in open arms & $1.17 \pm 0.24$ & $1.31 \pm 0.18$ & $1.55 \pm 0.31$ \\
Percent open arm entries & $29.61 \pm 3.82$ & $26.34 \pm 1.97$ & $28.24 \pm 3.44$ \\
Percent time spent in open arms & $13.17 \pm 2.63$ & $9.30 \pm 1.22$ & $10.68 \pm 2.18$ \\
Open field & & & $1.56 \pm 0.49$ \\
Total distance moved (m) & $111.09 \pm 8.92$ & $119.67 \pm 12.77$ & $117.77 \pm 10.38$ \\
Distance moved (m) in center zone & $7.99 \pm 0.88$ & $9.24 \pm 1.59$ & $9.16 \pm 0.96$ \\
Time in center zone (s) & $170.96 \pm 21.45$ & $200.04 \pm 38.23$ & $195.70 \pm 20.36$ \\
Center zone entries & $88 \pm 10$ & $106 \pm 19$ & $106 \pm 11$ \\
\hline
\end{tabular}

SAL-SAL, offspring subjected to prenatal vehicle exposure and raised by vehicle-treated surrogate mothers; SAL-POL, offspring subjected to prenatal vehicle exposure and raised by PolyI:C-treated surrogate mothers; POL-SAL, offspring subjected to prenatal PolyI:C exposure and raised by vehicle-treated surrogate mothers; POL-POL, offspring subjected to prenatal PolyI:C exposure and raised by PolyI:C-treated surrogate mothers. No significant group differences were detected in any of the dependent measures. All values are means \pm SEM 
the between-subjects factors of prenatal treatment or postnatal rearing were revealed in the ANOVA of total distance moved. Hence, the prenatal and postnatal manipulations did not significantly influence locomotor activity as assessed in the open field exploration test. The group means \pm SEM of all dependent measures in the open field test are provided in Table 2 .

Effects of the prenatal and postnatal manipulations on conditioned fear in peri-adolescence

\section{Conditioning}

The amount of freezing increased as a function of trials in all experimental groups as evident by the overall main effect of trials $\left(F_{2,64}=77.1, p<0.001\right)$ in the $2 \times 2 \times 2 \times 3$ (prenatal treatment $\times$ postnatal rearing $\times$ sex $\times$ trials) ANOVA of percent time freezing. The amount of freezing during acquisition of the conditioned freezing response was generally more pronounced in female animals compared to male animals, leading to a main effect of sex $\left(F_{1,32}=4.7\right.$, $p<0.05)$. Most importantly, being raised by a PolyI: C-challenged surrogate mother increased the amount of freezing in female offspring, and this effect was largely independent of the prenatal treatment histories. Hence, enhanced freezing was noted in female mice that were subjected to prenatal vehicle treatment and adopted by PolyI:C-treated surrogate mothers (i.e., SAL-POL females) as well as in female mice that were subjected to prenatal PolyI:C treatment and adopted by PolyI:C-treated surrogate mothers (i.e., POL-POL females) in comparison with the other two groups (i.e., SAL-SAL and POL-SAL offspring) (Fig. 3a). No similar effects of the postnatal rearing condition were observed in male offspring (Fig. 3a). This pattern of results led to a significant main effect of postnatal rearing $\left(F_{1,32}=5.9, p<0.05\right)$ and its interaction with sex $\left(F_{1,62}=5.7, p<0.05\right)$. No other main effects or interactions attained statistical significance.

Additional $2 \times 2 \times 3$ (prenatal treatment $\times$ postnatal rearing $\times$ trials) ANOVAs of percent time freezing restricted to both female and male subjects were then conducted. The analysis restricted to female animals confirmed that the adoption of prenatally PolyI:C or vehicle animals by PolyI:C-challenged surrogate mothers led to enhanced freezing in periadolescence, as indicated by the significant main effect of postnatal rearing $\left(F_{1,15}=5.7, p<0.05\right)$. The main effect of postnatal rearing was far from significant $(F<1.0$; n.s. $)$ in the ANOVA of percent time freezing restricted to male subjects.

\section{Context-freezing}

The expression of conditioned freezing towards the context in which conditioning took place (context A) was assessed when the animals were returned to the conditioning chamber $24 \mathrm{~h}$ following conditioning. Data were expressed as percent time freezing per 1-min bin and analyzed using a $2 \times 2 \times 2 \times 6$ (prenatal treatment $\times$ postnatal rearing $\times \operatorname{sex} \times$ 1 -min bin) ANOVA.

There was a general increase in the level of freezing as a function of time, leading to a significant main effect of bins $\left(F_{5,160}=2.4, p<0.05\right)$. The amount of context-freezing was generally more pronounced in female animals compared to male animals. ANOVA yielded a significant main effect of $\operatorname{sex}\left(F_{1,32}=4.7, p<0.05\right)$ and its interaction with bins $\left(F_{5,160}=\right.$ 2.9, $p<0.05)$. Similar to the results obtained in the conditioning phase, the adoption of neonates by surrogate mothers that had been exposed to PolyI:C treatment during pregnancy led to the emergence of enhanced freezing in female but not male peri-adolescent offspring (Fig. 3b). Again, this specific effect of the postnatal rearing condition was independent of the prenatal treatment histories: Increased context-freezing was evident in female mice that were subjected to prenatal vehicle treatment and adopted by PolyI:C-treated surrogate mothers (i.e., SAL-POL females) as well as in female subjects that were subjected to prenatal PolyI:C treatment and adopted by PolyI:C-treated surrogate mothers (i.e., POL-POL females) in comparison with the other two groups (i.e., SAL-SAL and POL-SAL offspring) (Fig. 3b). Statistical support for these impressions were yielded by the significant postnatal rearing $\times$ sex interaction $\left(F_{1,32}=4.4, p<0.05\right)$.

The subsequent $2 \times 2 \times 6$ (prenatal treatment $\times$ postnatal rearing $\times 1$-min bin) ANOVA of percent time freezing restricted to female subjects further confirmed that the adoption of prenatally PolyI:C or vehicle exposed subjects by PolyI:C-challenged surrogate mothers led to enhanced context-freezing in peri-adolescence, as indicated by the significant main effect of postnatal rearing $\left(F_{1,15}=4.2, p<\right.$ 0.05 ). On the other hand, the main effect of postnatal rearing was far from significant $(F<1.0 ;$ n.s. $)$ in the ANOVA restricted to male subjects, and no other main effects or interactions attained statistical significance in this analysis.

\section{Tone-freezing}

The expression of conditioned freezing towards the discrete tone CS was assessed in a novel context (context B) $48 \mathrm{~h}$ following conditioning. Data were expressed as percent time freezing per 1-min bin and analyzed using a $2 \times 2 \times 2 \times$ 6 (prenatal treatment $\times$ postnatal rearing $\times \operatorname{sex} \times 1$-min bin) ANOVA as described above.

Consistent with the postnatal maternal effects on contextfreezing (see Fig. 3b), the adoption by PolyI:C-challenged surrogate mothers increased the amount of freezing to the tone CS in female offspring (Fig. 3c). Again, this effect of 


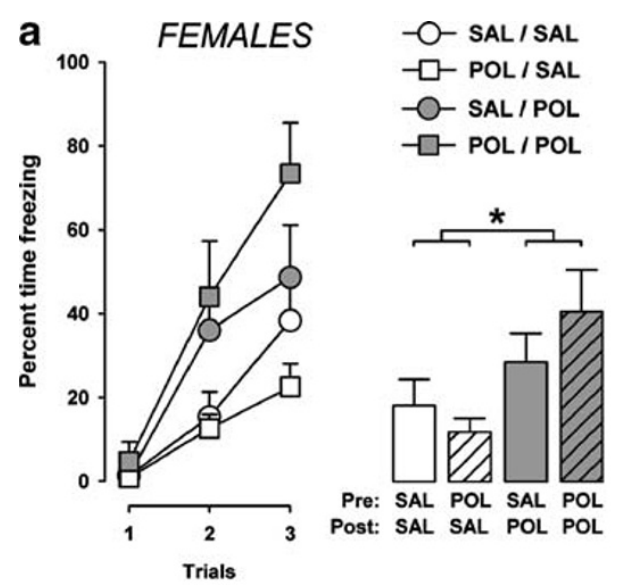

b

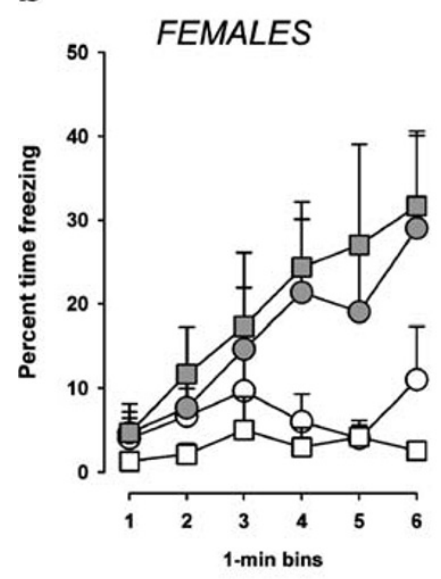

-O- SAL/SAL

$-\square-\mathrm{POL} / \mathrm{SAL}$

$-O-$ SAL/POL

$\rightarrow-\mathrm{POL} / \mathrm{POL}$

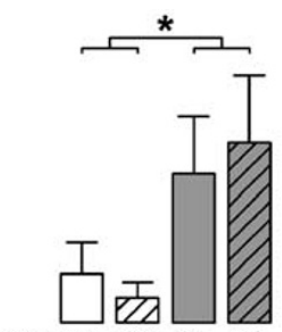

Pre: SAL POL SAL POL Post: SAL SAL POL POL

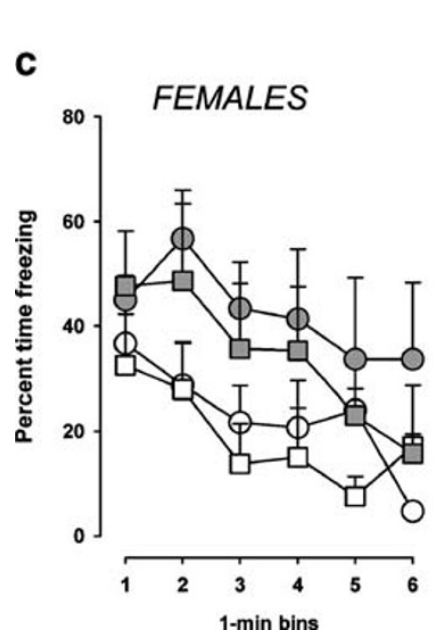

-O- SAL / SAL

$-\square-$ POL / SAL

-O- SAL/POL

$\neg-\mathrm{POL} / \mathrm{POL}$
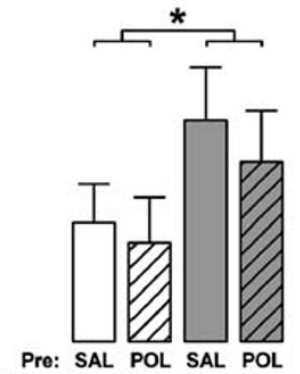

Post: SAL SAL POL POL

Fig. 3 Effects of the prenatal and postnatal maternal manipulations on the acquisition and expression of conditioned fear in the periadolescent offspring. a On the conditioning day, the amount of freezing (expressed as a percent of time freezing out of $30 \mathrm{~s}$ ) during presentations of the tone conditioned stimulus is depicted as a function of trials (line plots) and average over the three conditioning trials (bar plots) for both male and female subjects. b The amount of freezing (as a percent of time) exhibited over a period of 6 min when the animals were returned to the same context where tone-shock pairings took place $24 \mathrm{~h}$ earlier is illustrated as a function of 1-min bins (line plots) and as an average over the 6-min period (bar plots). $\mathbf{c}$ The amount of freezing (as a percent of time) displayed over a period of $6 \mathrm{~min}$ when
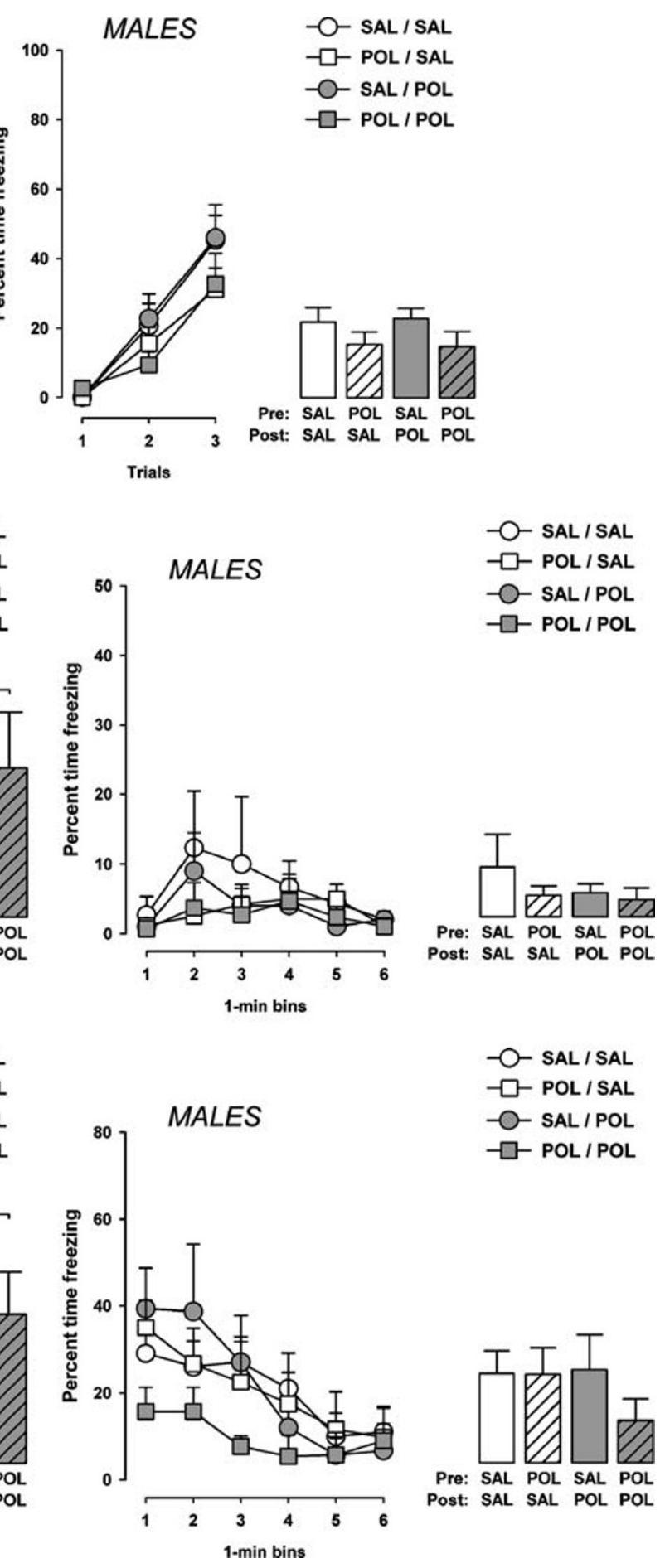

the animals were exposed to the same CS in a separate context $48 \mathrm{~h}$ following conditioning is illustrated as a function of 1-min bins (line plots) and as an average over the 6-min period (bar plots). SAL-SAL, offspring subjected to prenatal vehicle exposure and raised by vehicletreated surrogate mothers; SAL-POL, offspring subjected to prenatal vehicle exposure and raised by PolyI:C-treated surrogate mothers; POL-SAL, offspring subjected to prenatal PolyI:C exposure and raised by vehicle-treated surrogate mothers; POL-POL, offspring subjected to prenatal PolyI:C exposure and raised by PolyI:C-treated surrogate mothers. Symbol $(*)$ refers to a statistical significance of $p<0.05$. The number of subjects in each group is listed in Table 1 . All values are means \pm SEM 
the postnatal rearing condition was largely independent of the prenatal treatment histories and not seen in male offspring. Hence, enhanced tone-freezing was noted in female mice that were subjected to prenatal vehicle treatment and adopted by PolyI:C-treated surrogate mothers (i.e., SAL-POL females) as well as in female mice that were subjected to prenatal PolyI:C treatment and adopted by PolyI:C-treated surrogate mothers (i.e., POL-POL females) in comparison with the other two groups (i.e., SAL-SAL and POL-SAL offspring) (Fig. 3c). These impressions were supported by the significant main effect of sex $\left(F_{1,32}=5.1\right.$, $p<0.05)$ and its interaction with postnatal rearing $\left(F_{1,32}=\right.$ $4.6, p<0.05$ ), as well as by the subsequent $2 \times 2 \times 6$ (prenatal treatment $\times$ postnatal rearing $\times 1$-min bin) ANOVA restricted to female subjects revealing a significant main effect of postnatal rearing $\left(F_{1,15}=4.6, p<0.05\right)$.

As illustrated in Fig. 3c, male offspring subjected to prenatal PolyI:C exposure and raised by PolyI:C-treated surrogate mothers (i.e. male POL-POL offspring) tended to display a decreased conditioned freezing response to the tone CS in comparison with male offspring in the other experimental groups. However, the $2 \times 2 \times 6$ (prenatal treatment $\times$ postnatal rearing $\times 1$-min bin) ANOVA restricted to male subjects did not provide statistical support for this, as it did not reveal any significant main effect or interaction involving the between-subjects factor of prenatal treatment.

The conditioned freezing response generally decreased as a function of time. This extinction effect was evident in both male and female subjects regardless of the prenatal treatment and postnatal rearing conditions (Fig. 3c). This impression was supported by the significant main effect of 1 -min bins $\left(F_{5,160}=21.2, p<0.001\right)$ in the initial $2 \times 2 \times 2 \times 6$ (prenatal treatment $\times$ postnatal rearing $\times \operatorname{sex} \times$ 1 -min bin) ANOVA, as well as by the presence of significant main effects of 1-min bins in the subsequent $2 \times 2 \times 6$ (prenatal treatment $\times$ postnatal rearing $\times 1$-min bin) ANOVAs restricted to female and male subjects (females: $F_{5,75}=8.4, p<0.001$; males: $F_{5,85}=14.7, p<$ $0.001)$. There were no significant interactions between 1 -min bins and prenatal treatment or postnatal rearing in any of these analyses.

Effects of the prenatal and postnatal manipulations on locomotor activity and unconditioned anxiety-related behaviors in adulthood

\section{Elevated plus maze test}

Similar to the null effects in peri-adolescence (see Table 2), neither the prenatal nor postnatal maternal manipulations exerted any significant influence on anxiety-related behaviors in the adult offspring as assessed in the elevated plus maze. The dependent measures of percent time spent in the open arms, percent open arm entries, distance moved in the open arms, and total distance moved in the entire maze were highly comparable between the four experimental groups, as well as between sexes. The $2 \times 2 \times 2$ (prenatal treatment $\times$ postnatal rearing $\times$ sex) ANOVA of these measures revealed no significant main effects or interactions. The means \pm SEM of total distance moved, distance moved in the open arms, percent time spent in the open arms, and percent open arm entries for each experimental group are provided in Table 3.

Table 3 Summary of the effects of the prenatal and postnatal maternal manipulations on the offspring's performance in the elevated plus maze and open field exploration tests in adulthood

\begin{tabular}{|c|c|c|c|c|}
\hline \multirow[t]{2}{*}{ Experiment } & \multicolumn{4}{|c|}{ Experimental groups (prenatal treatment-postnatal rearing) } \\
\hline & SAL-SAL & SAL-POL & POL-SAL & POL-POL \\
\hline \multicolumn{5}{|l|}{ Elevated plus maze } \\
\hline Total distance moved $(\mathrm{m})$ & $9.56 \pm 0.56$ & $10.48 \pm 0.57$ & $11.34 \pm 0.64$ & $10.85 \pm 0.51$ \\
\hline Distance moved $(\mathrm{m})$ in open arms & $1.57 \pm 0.46$ & $1.25 \pm 0.22$ & $1.40 \pm 0.37$ & $1.16 \pm 0.29$ \\
\hline Percent open arm entries & $32.63 \pm 4.53$ & $31.39 \pm 3.96$ & $28.83 \pm 3.35$ & $31.57 \pm 3.86$ \\
\hline Percent time spent in open arms & $27.79 \pm 5.10$ & $20.76 \pm 3.55$ & $18.78 \pm 3.02$ & $17.52 \pm 2.98$ \\
\hline \multicolumn{5}{|l|}{ Open field } \\
\hline Total distance moved (m) & $123.71 \pm 3.95$ & $119.25 \pm 8.05$ & $112.55 \pm 12.42$ & $135.54 \pm 9.86$ \\
\hline Distance moved $(\mathrm{m})$ in center zone & $7.27 \pm 0.78$ & $7.56 \pm 1.25$ & $8.50 \pm 2.18$ & $8.62 \pm 1.48$ \\
\hline Time in center zone (s) & $137.72 \pm 21.29$ & $116.92 \pm 19.88$ & $163.40 \pm 57.76$ & $175.94 \pm 32.36$ \\
\hline Center zone entries & $68 \pm 7$ & $67 \pm 11$ & $77 \pm 19$ & $84 \pm 13$ \\
\hline
\end{tabular}

SAL-SAL, offspring subjected to prenatal vehicle exposure and raised by vehicle-treated surrogate mothers; SAL-POL, offspring subjected to prenatal vehicle exposure and raised by PolyI:C-treated surrogate mothers; POL-SAL, offspring subjected to prenatal PolyI:C exposure and raised by vehicle-treated surrogate mothers; POL-POL, offspring subjected to prenatal PolyI:C exposure and raised by PolyI:C-treated surrogate mothers. No significant group differences were detected in any of the dependent measures. All values are means \pm SEM 


\section{Open field exploration test}

Consistent with the findings obtained in peri-adolescent mice (see Table 2), the prenatal and postnatal maternal manipulations did not significantly influence the offspring's exploratory behavior in the open field test when assessed in adulthood. The $2 \times 2 \times 2 \times 6$ (prenatal treatment $\times$ postnatal rearing $\times$ sex $\times 10$-min bins) ANOVAs of center zone entries, time spent in the center zone, distance moved in the center zone, and total distance traveled in the entire open field did not reveal any significant main effects or interactions involving the between-subjects factors of prenatal treatment and postnatal rearing. Furthermore, these measures were also largely comparable between adult male and female subjects: ANOVA did not obtain any significant main effects or interactions involving the factor of sex. There was a clear habituation effect in all experimental groups, with center zone entries, time spent in the center zone, distance moved in the center zone, and total distance traveled in the entire arena decreasing over time. This led to a significant main effect of 10-min bins in the ANOVAs of these measures (center zone entries: $F_{5,160}=28.9, p<0.001$; time spent in the center zone: $F_{5,160}=4.6, p<0.001$; distance moved in the center zone: $F_{5,160}=32.2, p<0.001$; total distance traveled: $\left.F_{5,160}=117.9, p<0.001\right)$. The means \pm SEM of all independent variables analyzed in the open field exploration test at adult age are provided in Table 3.

Effects of the prenatal and postnatal manipulations on conditioned fear in adulthood

\section{Conditioning}

The amount of freezing increased as a function of trials in all experimental groups as evident by the overall main effect of trials $\left(F_{2,64}=85.8, p<0.001\right)$ in the $2 \times 2 \times 2 \times 3$ (prenatal treatment $\times$ postnatal rearing $\times$ sex $\times$ trials) ANOVA of percent time freezing. Consistent with the results obtained in peri-adolescent subjects, the amount of freezing during acquisition of the conditioned freezing response was generally more pronounced in female animals compared to male animals (Fig. 4a), leading to a main effect of sex $\left(F_{1,32}=5.2, p<0.05\right)$ and its interaction with trials $\left(F_{2,64}=7.1, p<0.001\right)$. Subsequent $2 \times 2 \times 3$ (prenatal treatment $\times$ postnatal rearing $\times$ trials) ANOVAs restricted to female and male subjects were then performed in order to analyze the influence of the prenatal and postnatal maternal manipulations on acquisition of the conditioned freezing response in either sex. The ANOVA of percent time freezing restricted to male animals did not reveal any significant main effects or interactions involving the between-subjects factors of prenatal treatment and postnatal rearing. Hence, the acquisition of the conditioned freezing response in the adult male offspring was not affected by the prenatal and postnatal manipulations (Fig. 4a).

In contrast, female offspring born to PolyI:C-treated mothers displayed a significant reduction in percent time freezing during conditioning compared to females born to mothers exposed to vehicle treatment during pregnancy. This effect emerged regardless of the postnatal rearing condition. Therefore, reduced percent time freezing was noted in female mice that were subjected to prenatal PolyI: $\mathrm{C}$ treatment and adopted by PolyI:C-treated surrogate mothers (i.e., POL-POL females) as well as in female mice that were subjected to prenatal PolyI:C treatment and adopted by vehicle-treated surrogate mothers (i.e., POLSAL females) in comparison with the other two groups (i.e., SAL-SAL and SAL-POL offspring) (Fig. 4a). Statistical support for these impressions was yielded by the $2 \times$ $2 \times 3$ (prenatal treatment $\times$ postnatal rearing $\times$ trials) ANOVA restricted to female offspring, which revealed a significant main effect of prenatal treatment $\left(F_{1,15}=4.7, p<\right.$ $0.05)$ and its interaction with trials $\left(F_{1,15}=3.9, p<0.05\right)$. No other main effects or interactions attained statistical significance.

\section{Context-freezing}

The expression of conditioned freezing towards the context in which conditioning took place (context A) was assessed when the animals were returned to the conditioning chamber $24 \mathrm{~h}$ following conditioning. Data were expressed as percent time freezing per 1-min bin and analyzed using a $2 \times 2 \times 2 \times 6$ (prenatal treatment $\times$ postnatal rearing $\times \operatorname{sex} \times$ 1-min bin) split-plot ANOVA as described before.

Conditioned context-freezing was generally more pronounced in female animals compared to male animals (Fig. 4b), as supported by the main effect of $\operatorname{sex}\left(F_{1,15}=\right.$ 9.7, $p<0.01)$. Consistent with postnatal maternal effects on context-freezing in peri-adolescent mice (see Fig. 3b), the adoption of neonates by surrogate mothers that had been exposed to PolyI:C treatment during pregnancy led to the emergence of enhanced freezing in female but not male offspring at adult age (Fig. 4b). Again, this effect of the postnatal rearing condition was independent of the prenatal treatment histories: Increased context-freezing was evident in adult female mice that were subjected to prenatal vehicle treatment and adopted by PolyI:C-treated surrogate mothers (i.e., SAL-POL females) as well as in female subjects that were subjected to prenatal PolyI:C treatment and adopted by PolyI:C-treated surrogate mothers (i.e., POL-POL females) in comparison with the other two groups (i.e., SAL-SAL and POL-SAL offspring) (Fig. 4b). Statistical support for this interpretation was yielded by the significant main effect of postnatal rearing $\left(F_{1,32}=5.5, p<0.05\right)$ and its interaction with $\operatorname{sex}\left(F_{5,160}=4.2, p<0.05\right)$, as well as by the subsequent 

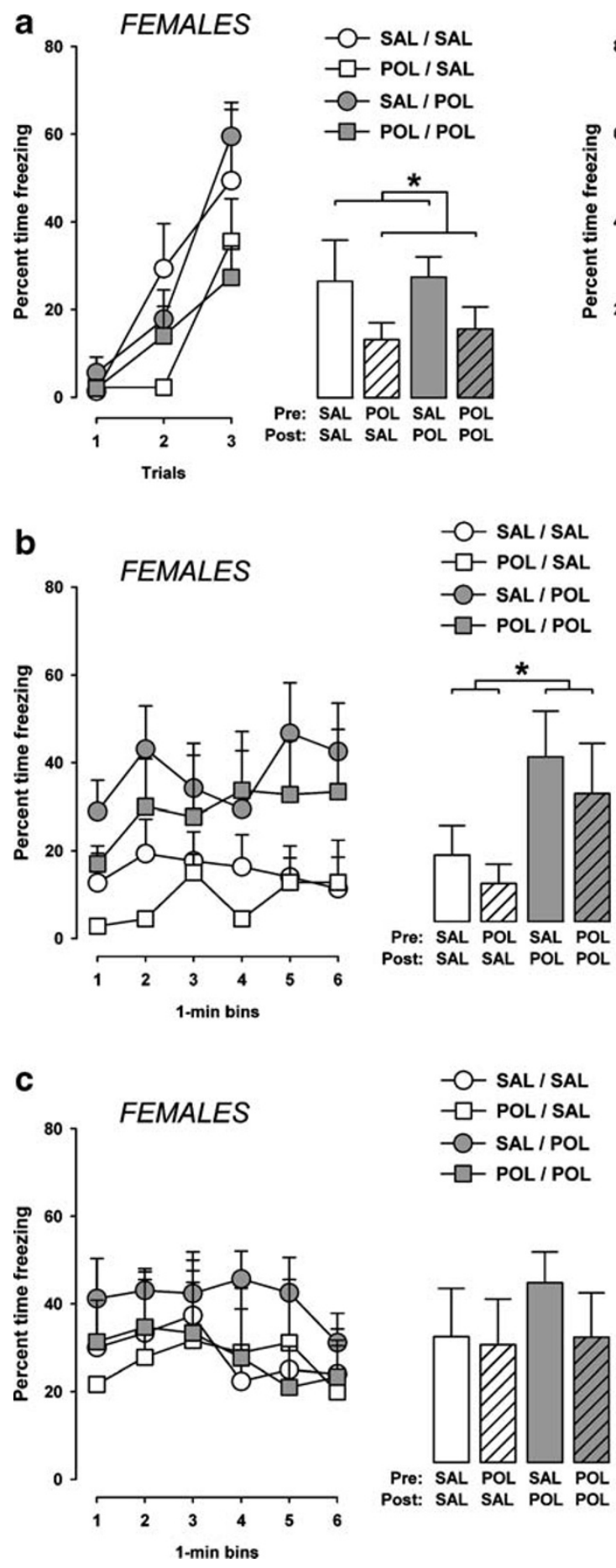

Fig. 4 Effects of the prenatal and postnatal maternal manipulations on the acquisition and expression of conditioned fear in the adult offspring. a On the conditioning day, the amount of freezing (expressed as a percent of time freezing out of $30 \mathrm{~s}$ ) during presentations of the tone conditioned stimulus is depicted as a function of trials (line plots) and average over the three conditioning trials (bar plots) for both male and female subjects. b The amount of freezing (as a percent of time) exhibited over a period of 6 min when the animals were returned to the same context where tone-shock pairings took place $24 \mathrm{~h}$ earlier is illustrated as a function of 1-min bins (line plots) and as an average over the 6-min period (bar plots). $\mathbf{c}$ The amount of freezing (as a percent of time) displayed over
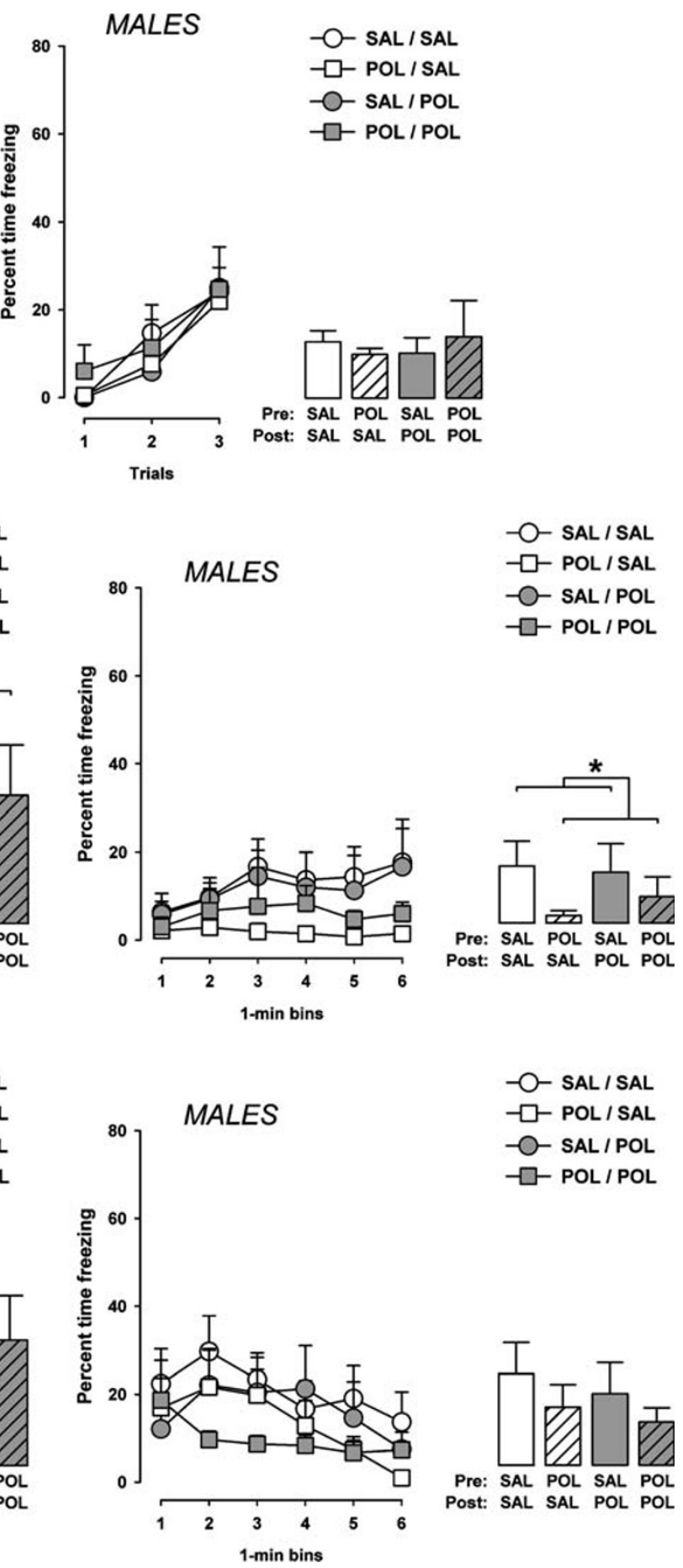

a period of 6 min when the animals were exposed to the same CS in a separate context $48 \mathrm{~h}$ following conditioning is illustrated as a function of 1-min bins (line plots) and as an average over the 6-min period (bar plots). SAL-SAL, offspring subjected to prenatal vehicle exposure and raised by vehicle-treated surrogate mothers; SAL-POL, offspring subjected to prenatal vehicle exposure and raised by PolyI: C-treated surrogate mothers; POL-SAL, offspring subjected to prenatal PolyI:C exposure and raised by vehicle-treated surrogate mothers; POL-POL, offspring subjected to prenatal PolyI:C exposure and raised by PolyI:C-treated surrogate mothers. Symbol $(*)$ refers to a statistical significance of $p<0.05$. The number of subjects in each group is listed in Table 1 . All values are means \pm SEM 
$2 \times 2 \times 6$ (prenatal treatment $\times$ postnatal rearing $\times 1$-min bin) ANOVA of percent time freezing restricted to female subjects revealing significance for the main effect of postnatal rearing $\left(F_{1,15}=4.6, p<0.05\right)$.

On the other hand, maternal PolyI:C-treatment during pregnancy led to a small but significant decrease in the conditioned context-freezing response in adult male subjects. Expression of the conditioned freezing response towards the context was lowest in male animals exposed to prenatal PolyI:C treatment and adopted by vehicletreated surrogate mothers (i.e., POL-SAL males), followed by male mice that were subjected to prenatal PolyI:C treatment and adopted by PolyI:C-treated surrogate mothers (i.e., POL-POL mice) (Fig. 4b). Conditioned contextfreezing was highly comparable in prenatally vehicleexposed offspring that were adopted by either PolyI:C- or vehicle-treated surrogate mothers (Fig. 4b). This pattern of results led to a significant main effect of prenatal treatment $\left(F_{1,17}=4.9, p<0.05\right)$ in the $2 \times 2 \times 6$ (prenatal treatment $\times$ postnatal rearing $\times 1$-min bin) ANOVA of percent time freezing restricted to male subjects, with no other main effects or interactions attaining statistical significance.

\section{Tone-freezing}

The expression of conditioned freezing towards the discrete tone CS was assessed in a novel context (context B) $48 \mathrm{~h}$ following conditioning as described before. Data were expressed as percent time freezing per 1-min bin and analyzed using a $2 \times 2 \times 2 \times 6$ (prenatal treatment $\times$ postnatal rearing $\times \operatorname{sex} \times 1$-min bin) split-plot ANOVA.

In contrast to the findings obtained in peri-adolescent mice (see Fig. 3c), the adoption of neonates by surrogate rearing mothers that been exposed to PolyI:C exposure during pregnancy did not significantly influence the adult offspring's conditioned response to the tone CS (Fig. 4c). Conditioned tone-freezing was also not significantly affected by the prenatal immunological manipulation (Fig. 4c). ANOVA of percent time freezing only revealed significance for the main effects of sex $\left(F_{1,32}=15.0, p<0.001\right)$ and bins $\left(F_{5,160}=7.9, p<0.001\right)$. The former reflects the higher level of tone-freezing in female animals compared to male animals, and the latter indicates the emergence of extinction of the conditioned tone-freezing response over time regardless of the experimental manipulations and sex (Fig. 4c).

\section{Discussion}

The present study demonstrates that maternal immune activation during pregnancy leads to altered postpartum maternal behavior in the form of reduced licking/ grooming of pups and increased nest building activity.
These alterations in maternal behavior were paralleled by postnatal maternal effects on the development of abnormal fear-related behavior in the offspring: The adoption of neonates by PolyI:C-challenged surrogate rearing mothers led to enhanced conditioned fear in the periadolescent and adult offspring. These postnatal maternal influences on fear-related behavior were independent of the prenatal treatment histories, because increased conditioned fear emerged in offspring adopted by PolyI:Cchallenged surrogate mothers regardless of whether the neonates were prenatally exposed to PolyI:C or vehicle treatment. The null effects of prenatal PolyI:C-induced immune activation and adoption by immune-challenged surrogate mothers in the elevated plus maze and open field exploration tests suggests that the prenatal and postnatal maternal manipulations did not exert any noticeable influences on unconditioned (innate) anxiety-related behavior in the offspring [4].

Increased fear-related behavior was exclusively seen in the female but not male subjects. This suggests that female offspring may be more sensitive to the negative influences of immuno-precipitated deficits in maternal care on subsequent behavioral development in comparison with male offspring. A strikingly similar conclusion can also be drawn from numerous experimental studies designed to investigate the impact of maternal exposure to stressful events during pregnancy on anxiety- and depression-like behavior in the offspring (for a recent review see [70]. Specifically, increased anxiety-, fear- and depression-like behavior is more readily seen in prenatally stressed female offspring, whilst abnormal cognitive development is more prevalent in male offspring born to gestationally stressed mothers [70]. However, if exposure to prenatal stress resulting from immunological stimulation during pregnancy might indeed account for the development of abnormal fear-related behavior as seen in our study, this effect would most likely be attributable to postnatal rather than prenatal maternal effects on the offspring.

The alterations in conditioned fear emerging in female offspring reared by PolyI:C-challenged surrogate mothers were arguably more pronounced when assessed in periadolescence as compared to adulthood. This is because in peri-adolescence, all critical measures of conditioned fear were affected by the postnatal adoption procedure, namely the acquisition of the conditioned fear response (Fig. 3a), as well as the expression of conditioned fear towards the context (Fig. 3b) and the tone CS (Fig. 3c). On the contrary, female offspring reared by PolyI:C-exposed surrogate mothers displayed a specific enhancement of conditioned fear towards the context when they were tested in adulthood (Fig. 4b). It follows that deficient maternal care resulting from immune challenge during pregnancy may have a more extensive impact on fear-related behavior in 
peri-adolescent relative to adult female offspring. However, the adoption-induced disturbances in the expression of conditioned fear towards a context, in which aversive associations were formed, appear to be long-lasting and persist from the peri-adolescent to the adult stage of development. Interestingly, the acquisition of the conditioned freezing response was retarded in adult subjects born to PolyI:C-treated mothers. Again, this effect was sexdependent and only emerged in female but not male subjects (Fig. 4a). This is contrary to the adoption-induced effects on the acquisition of the conditioned freezing response in pre-adolescence (see Fig. 4a). One implication form this contrast is that the specificity of abnormalities in acquiring fear-related behavior is critically influenced by the postnatal stage of development.

Whilst there were no significant prenatal maternal effects on anxiety- and fear-related behavior in peri-adolescent offspring, prenatal immune challenge led to a small but significant reduction in the expression of conditioned contextual fear in adult male subjects. This effect was independent of the postnatal rearing condition (Fig. 4b). Hence, in addition to the influence of the postnatal rearing condition, alterations in the expression of conditioned fear can also be induced by inflammatory processes taking place in prenatal life. Notably, the prenatal and postnatal manipulations exerted opposite effects on the offspring's conditioned fear response in two ways: First, in utero exposure to immune challenge and adoption by immunechallenged surrogate mothers led to a decrease and an enhancement of conditioned fear, respectively. Second, whilst being reared by immune-challenged surrogate mothers can induce both pre- and post-pubertal changes in the acquisition and/or development of conditioned fear, the alterations in conditioned fear induced by prenatal exposure to immune challenge appear to be dependent on postpubertal maturational processes. This latter effect is in full agreement with the previous observations that the full spectrum of prenatal PolyI:C-induced behavioral, cognitive, and pharmacological abnormalities in rats and mice emerges only after the post-pubertal stage of development $[40,42,48,72,73]$.

It is well known that mother-infant interactions are reciprocal $[20,68]$. The rearing mother's care influences the physiological and neurobiobehavioral development of the offspring, while in turn the offspring's affiliative behavior and demands critically determine how competent maternal care will be. In our cross-fostering design, each surrogate mother concomitantly raised pups of both prenatal treatment conditions. This ensured that each surrogate mother was exposed to similar affiliative behaviors and demands originating from the pups. Therefore, we can rule out the possibility that the changes in maternal behavior observed in PolyI:C-challenged surrogate mothers may result from possible differences in affiliative behavior and/or demands between neonates born to PolyI:C-treated and control mothers [45]. Rather, the identified deficits in maternal care are likely to be accounted for by the inflammationassociated processes taking place in late gestation. As already mentioned in the introduction, viral-like immunological stimulation is strongly linked to the activation of several stress response systems, including the HPA axis [23, 44, 69]. Consistent with previous reports (e.g., [34, 50, 63], the induction of maternal stress responses may thus represent a plausible mechanism by which postpartum maternal behavior could be affected by immunological stimulation during pregnancy. We did not find any body weight changes in offspring raised by surrogate mothers that had been exposed to PolyI:C-induced immune activation during pregnancy. This indicates that PolyI:C-induced immunological stress during pregnancy may not have affected the quantity and/or quality of milk production, which is essential for successful postnatal growth and development [47]. It is also unlikely that prenatal PolyI:C exposure could have led to persistent inflammation in the maternal peripheral immune system and cytokines in the milk, because a single administration of PolyI:C is known to result in a time-limited elevation of cytokines in the host (see e.g., [16, 21, 39].

Among the various components of postpartum maternal behavior, the immunological manipulation in late gestation most clearly affected maternal licking/grooming and nest building (Fig. 2). Individual differences among lactating rats in the frequency of maternal licking/grooming have long been recognized to exert a critical influence on the offsprings physiological and neurobiobehavioral development [7, 8, 27, 28, 29]. For example, adult rats reared by mothers that naturally exhibit reduced pup licking/grooming behavior show increased behavioral and endocrine responses to stress in comparison with offspring of mothers that exhibit high rates of pup licking/grooming [8, 29, 35]. Most importantly, offspring of mothers with reduced pup licking/grooming behavior display enhanced fear reactivity in the form of increased shock-induced freezing behavior compared to offspring raised by mothers with high rates of pup licking/ grooming [35]. Immunologically induced deficits in maternal licking/grooming behavior may thus also represent an important postnatal maternal factor for the precipitation of abnormal fear-related behavior in the fostered offspring as identified here. However, naturally occurring deficits in maternal licking/grooming appear to affect fear-related behavior in the offspring regardless of sex [8, 10, 29, 35]. This is in contrast with the present findings, which indicate that deficient maternal care resulting from immune activation during pregnancy preferentially affects fear-related behavior in the female but not male offspring. It needs to be further explored whether this apparent discrepancy may be attribut- 
able to differences in the experimental design (i.e., immunologically induced versus naturally occurring variations in maternal licking/grooming) and/or differences in the choice of the rodent species (mice versus rats).

As reviewed in detail elsewhere [37, 43], various morphological, neuroanatomical, and neurochemical effects can be identified in rats and mice subjected to in utero immune activation. The neuropathological deficits induced by prenatal immune challenge include pre- and postsynaptic changes in various neurotransmitter systems such as the central dopamine, $\gamma$-aminobutyric acid (GABA), glutamate, and serotonin systems, together with alterations in neuronal and glial cell number, structure and positioning. At least some of these changes are accounted for by prenatal rather than postnatal maternal effects on the offspring, because they emerge in prenatally immune challenged animals regardless of whether they are raised by control surrogate mothers or by gestationally immune-challenged surrogate mothers [40]. In contrast to the identified prenatal maternal contributions to abnormal postnatal brain structure and function, it remains largely unexplored to date whether changes in postnatal maternal factors resulting from immunological stress during pregnancy can also induce multiple neuropathological signs in the offspring. At the present stage, we can thus only speculate about the neural and/or endocrinological mechanisms underlying the emergence of enhanced fear-related behavior following adoption by rearing mothers that were exposed to immune activation during pregnancy.

One clear possibility would be that deficient maternal care resulting from immune challenge during pregnancy negatively influences the offspring's development of brain structures crucially involved in the acquisition and/or expression of conditioned fear, especially the amygdala and hippocampus [19, 26, 31]. The amygdala is well known for playing an essential role not only in the acquisition of conditioned freezing behavior, but also in the expression of conditioned fear towards the discrete CS [19, 26, 32]. On the other hand, the hippocampal formation appears to provide the amygdala with information about contextual cues and is thus essential for the consolidation and expression of contextual fear $[1,19]$. One expectation from the present results would be that the adoption of neonates by immune-challenged surrogate mothers may lead to concomitant amygdalar and hippocampal dysfunctions in the peri-adolescent offspring, because all critical measures of conditioned fear were affected at this stage of development (Fig. 3). In contrast, adult offspring raised by PolyI:Cexposed surrogate mothers may display abnormalities preferentially in the hippocampal formation, because they showed a specific enhancement of conditioned fear towards the context when they were tested in adulthood (Fig. 4b).

One alternative (but not mutually exclusive) possibility would be that the adoption by gestationally immune- challenged mothers interferes with the normal development of the offspring's stress response system, including the HPA axis. In fact, numerous studies support a crucial role of the HPA axis in the expression of conditioned fear, especially towards contextual cues $[14,15,18,25,54,60]$. Additional studies are thus clearly warranted in order to identify possible neuroendocrinological mechanisms underlying the emergence of enhanced fear-related behavior in offspring reared by mothers that had experienced immune activation during pregnancy.

In conclusion, our results provide experimental evidence that the adoption of neonates by surrogate mothers that were exposed to immunological stimulation during pregnancy increases vulnerability for fear-related pathology in the fostered offspring, an effect that appears to be linked to changes in postpartum maternal behavior. Hence, immunological stress during pregnancy represents a significant environmental risk factor for abnormal postnatal maternal care and subsequent behavioral disorders in the offspring. Abnormal fear-related behavior is implicated in a variety of neuropsychiatric disorders, including panic disorder, phobia, post-traumatic stress disorder, and obsessive-compulsive disorder [25, 33]. Our findings may thus also have important implications for the identification of etiopathological mechanisms involved in the development of neuropsychiatric disorders that are characterized by fearassociated psychopathology.

Acknowledgments We are extremely grateful to Peter Schmid for his technical assistance and to Flavia May for running parts of the behavioral experiments. We also remain indebted to the Animal Services Department Schwerzenbach, Swiss Federal Institute of Technology, for their excellent animal husbandry and care and to Natalie Aeschbach-Jones for her editorial assistance. This work was supported by the Swiss Federal Institute of Technology Zurich (ETHZ) and the Swiss National Science Foundation (SNSF). ETHZ and SNSF had no further role in the design of the study, in the collection, analysis and interpretation of the data, in writing the report, or in the decision to submit the paper for publication.

Conflict of interest statement All authors declare to have no conflicts of interest.

\section{References}

1. Anagnostaras SG, Gale GD, Fanselow MS. Hippocampus and contextual fear conditioning: recent controversies and advances. Hippocampus 2001;11:8-17.

2. Arndt TL, Stodgell CJ, Rodier PM. The teratology of autism. Int J Dev Neurosci. 2005;23:189-99.

3. Ashdown H, Dumont Y, Ng M, Poole S, Boksa P, Luheshi GN. The role of cytokines in mediating effects of prenatal infection on the fetus: implications for schizophrenia. Mol Psychiatry. 2006;11:47-55.

4. Belzung C, Griebel G. Measuring normal and pathological anxiety-like behaviour in mice: a review. Behav Brain Res. 2001; 125:141-49. 
5. Brown AS. Prenatal infection as a risk factor for schizophrenia. Schizophr Bull. 2006;32:200-2.

6. Brown AS, Susser ES. In utero infection and adult schizophrenia. Ment Retard Dev Disabil Res Rev. 2002;8:51-7.

7. Caldji C, Diorio J, Meaney MJ. Variations in maternal care alter GABA(A) receptor subunit expression in brain regions associated with fear. Neuropsychopharmacology 2003;28:1950-9.

8. Caldji C, Tannenbaum B, Sharma S, Francis D, Plotsky PM, Meaney MJ. Maternal care during infancy regulates the development of neural systems mediating the expression of fearfulness in the rat. Proc Natl Acad Sci U S A. 1998;95:5335-40.

9. Champagne FA, Francis DD, Mar A, Meaney MJ. Variations in maternal care in the rat as a mediating influence for the effects of environment on development. Physiol Behav. 2003;79:359-71.

10. Champagne FA, Meaney MJ. Stress during gestation alters postpartum maternal care and the development of the offspring in a rodent model. Biol Psychiatry. 2006;59:1227-35.

11. Chess S. Autism in children with congenital rubella. J Autism Child Schizophr. 1971;1:33-47.

12. Cirulli F, Berry A, Alleva E. Early disruption of the mother-infant relationship: effects on brain plasticity and implications for psychopathology. Neurosci Biobehav Rev. 2003;27:73-82.

13. Clancy B, Darlington RB, Finlay BL. Translating developmental time across mammalian species. Neuroscience 2001;105:7-17.

14. Cordero MI, Merino JJ, Sandi C. Correlational relationship between shock intensity and corticosterone secretion on the establishment and subsequent expression of contextual fear conditioning. Behav Neurosci. 1998;112:885-91.

15. Cordero MI, Sandi CA. A role for brain glucocorticoid receptors in contextual fear conditioning: dependence upon training intensity. Brain Res. 1998;786:11-7.

16. Cunningham C, Campion S, Teeling J, Felton L, Perry VH. The sickness behaviour and CNS inflammatory mediator profile induced by systemic challenge of mice with synthetic doublestranded RNA (poly I:C). Brain Behav Immun. 2007;21:490-502.

17. Dammann O, Kuban KC, Leviton A. Perinatal infection, fetal inflammatory response, white matter damage, and cognitive limitations in children born preterm. Ment Retard Dev Disabil Res Rev. 2002;8:46-50.

18. de Kloet ER, Oitzl MS, Joëls M. Stress and cognition: are corticosteroids good or bad guys? Trends Neurosci. 1999;22:422-6.

19. Fendt M, Fanselow MS. The neuroanatomical and neurochemical basis of conditioned fear. Neurosci Biobehav Rev. 1999;23:743-60.

20. Fleming AS, O’Day DH, Kraemer GW. Neurobiology of motherinfant interactions: experience and central nervous system plasticity across development and generations. Neurosci Biobehav Rev. 1999;23:673-85.

21. Fortier ME, Kent S, Ashdown H, Poole S, Boksa P, Luheshi GN. The viral mimic, polyinosinic:polycytidylic acid, induces fever in rats via an interleukin-1-dependent mechanism. Am J Physiol Regul Integr Comp Physiol. 2004;287:R759-66.

22. Gilmore JH, Jarskog LF. Exposure to infection and brain development: cytokines in the pathogenesis of schizophrenia. Schizophr Res. 1997;24:365-7.

23. Haddad JJ, Saadé NE, Safieh-Garabedian B. Cytokines and the neuro-immune-endocrine interactions: a role for the hypothalamicpituitary-adrenal revolving axis. J Neuroimmunol. 2002;133:1-19.

24. Kaufman MH. The atlas of mouse development. London: Academic; 2003.

25. Korte SM. Corticosteroids in relation to fear, anxiety and psychopathology. Neurosci Biobehav Rev. 2001;25:117-42.

26. LeDoux JE. Emotion circuits in the brain. Annu Rev Neurosci. 2000;23:155-84.

27. Levine S. Primary social relationships influence the development of the hypothalamic-pituitary-adrenal axis in the rat. Physiol Behav. 2001;73:255-60.
28. Liu D, Diorio J, Day JC, Francis DD, Meaney MJ. Maternal care, hippocampal synaptogenesis and cognitive development in rats. Nat Neurosci. 2000;3:799-806.

29. Liu D, Diorio J, Tannenbaum B, Caldji C, Francis D, Freedman A, Sharma S, Pearson D, Plotsky PM, Meaney MJ. Maternal care, hippocampal glucocorticoid receptors, and hypothalamic-pituitaryadrenal responses to stress. Science 1997;277:1659-62.

30. Macrí S, Mason GJ, Würbel H. Dissociation in the effects of neonatal maternal separations on maternal care and the offspring's HPA and fear responses in rats. Eur J Neurosci. 2004;20:1017-24.

31. Maren S. Neurobiology of Pavlovian fear conditioning. Annu Rev Neurosci. 2001;24:897-931.

32. Maren S, Quirk GJ. Neuronal signalling of fear memory. Nat Rev Neurosci. 2004;5:844-52.

33. McNaughton N, Corr PJ. A two-dimensional neuropsychology of defense: fear/anxiety and defensive distance. Neurosci Biobehav Rev. 2004;28:285-305.

34. Meek LR, Dittel PL, Sheehan MC, Chan JY, Kjolhaug SR. Effects of stress during pregnancy on maternal behavior in mice. Physiol Behav. 2001;72:473-9.

35. Menard JL, Champagne DL, Meaney MJ. Variations of maternal care differentially influence 'fear' reactivity and regional patterns of cFos immunoreactivity in response to the shock-probe burying test. Neuroscience 2004;129:297-308.

36. Meyer U, Feldon J, Schedlowski M, Yee BK. Towards an immuno-precipitated neurodevelopmental animal model of schizophrenia. Neurosci Biobehav Rev. 2005;29:913-47.

37. Meyer U, Feldon J, Yee BK. A review of the fetal brain cytokine imbalance hypothesis of schizophrenia. Schizophr Bull. 2008a; in press.

38. Meyer U, Murray PJ, Urwyler A, Yee BK, Schedlowski M, Feldon J. Adult behavioral and pharmacological dysfunctions following disruption of the fetal brain balance between proinflammatory and IL-10-mediated anti-inflammatory signaling. Mol Psychiatry. 2008b;13:208-21.

39. Meyer U, Nyffeler M, Engler A, Urwyler A, Schedlowski M, Knuesel I, Yee BK, Feldon J. The time of prenatal immune challenge determines the specificity of inflammation-mediated brain and behavioral pathology. J Neurosci 2006a;26:4752-62.

40. Meyer U, Nyffeler M, Schwendener S, Knuesel I, Yee BK, Feldon J. Relative prenatal and postnatal maternal contributions to schizophrenia-related neurochemical dysfunction after in utero immune challenge. Neuropsychopharmacology 2008c;33:441-56.

41. Meyer U, Nyffeler M, Yee BK, Knuesel I, Feldon J. Adult brain and behavioral pathological markers of prenatal immune challenge during early/middle and late fetal development in mice. Brain Behav Immun. 2008d;22:469-86.

42. Meyer U, Schwendener S, Feldon J, Yee BK. Prenatal and postnatal maternal contributions in the infection model of schizophrenia. Exp Brain Res 2006b;173:243-57.

43. Meyer U, Yee BK, Feldon J. The neurodevelopmental impact of prenatal infections at different times of pregnancy: the earlier the worse? Neuroscientist 2007;13:241-56.

44. Milton NG, Hillhouse EW, Milton AS. Activation of the hypothalamo-pituitary-adrenocortical axis in the conscious rabbit by the pyrogen polyinosinic:polycytidylic acid is dependent on corticotrophin-releasing factor-41. J Endocrinol. 1992;135:69-75.

45. Moore CL, Power KL. Prenatal stress affects mother-infant interaction in Norway rats. Dev Psychobiol. 1986;19:235-45.

46. Nawa H, Takei N. Recent progress in animal modeling of immune inflammatory processes in schizophrenia: implication of specific cytokines. Neurosci Res. 2006;56:2-13.

47. Oken E, Lightdale JR. Updates in pediatric nutrition. Curr Opin Pediatr. 2000;12:282-90.

48. Ozawa K, Hashimoto K, Kishimoto T, Shimizu E, Ishikura H, Iyo M. Immune activation during pregnancy in mice leads to 
dopaminergic hyperfunction and cognitive impairment in the offspring: a neurodevelopmental animal model of schizophrenia. Biol Psychiatry. 2006;59:546-554.

49. Parker G, Hadzi-Pavlovic D, Greenwald S, Weissman M. Low parental care as a risk factor to lifetime depression in a community sample. J Affect Disord. 1995;33:173-80.

50. Patin V, Lordi B, Vincent A, Thoumas JL, Vaudry H, Caston J. Effects of prenatal stress on maternal behavior in the rat. Brain Res Dev Brain Res. 2002;139:1-8.

51. Patterson PH. Maternal infection: window on neuroimmune interactions in fetal brain development and mental illness. Curr Opin Neurobiol. 2002;12:115-8.

52. Patterson PH. Neuroscience. Maternal effects on schizophrenia risk. Science 2007;318:576-7.

53. Pryce CR, Feldon J. Long-term neurobehavioural impact of the postnatal environment in rats: manipulations, effects and mediating mechanisms. Neurosci Biobehav Rev. 2003;27:57-71.

54. Pugh CR, Tremblay D, Fleshner M, Rudy JW. A selective role for corticosterone in contextual-fear conditioning. Behav Neurosci. 1997;111:503-11

55. Rasier G, Toppari J, Parent AS, Bourguignon JP. Female sexual maturation and reproduction after prepubertal exposure to estrogens and endocrine disrupting chemicals: a review of rodent and human data. Mol Cell Endocrinol. 2006;254-255:187-201.

56. Rees S, Harding R. Brain development during fetal life: influences of the intra-uterine environment. Neurosci Lett. 2004; 361:111-4.

57. Rees S, Inder T. Fetal and neonatal origins of altered brain development. Early Hum Dev. 2005;81:753-61.

58. Richmond MA, Murphy CA, Pouzet B, Schmid P, Rawlins JN, Feldon J. A computer controlled analysis of freezing behaviour. J Neurosci Methods. 1998;86:91-9.

59. Rüedi-Bettschen D, Feldon J, Pryce CR. Circadian- and temperature-specific effects of early deprivation on rat maternal care and pup development: short-term markers for long-term effects? Dev Psychobiol. 2004;45:59-71.

60. Schulkin J, Morgan MA, Rosen JB. A neuroendocrine mechanism for sustaining fear. Trends Neurosci. 2005;28:629-35.

61. Shi L, Fatemi SH, Sidwell RW, Patterson PH. Maternal influenza infection causes marked behavioral and pharmacological changes in the offspring. J Neurosci. 2003;23:297-302.

62. Sisk CL, Foster DL. The neural basis of puberty and adolescence. Nat Neurosci. 2004;7:1040-47.
63. Smith JW, Seckl JR, Evans AT, Costall B, Smythe JW. Gestational stress induces post-partum depression-like behaviour and alters maternal care in rats. Psychoneuroendocrinology 2004;29:227-44.

64. Smith SE, Li J, Garbett K, Mirnics K, Patterson PH. Maternal immune activation alters fetal brain development through interleukin-6. J Neurosci. 2007;27:10695-702.

65. Spear LP. The adolescent brain and age-related behavioral manifestations. Neurosci Biobehav Rev. 2000;24:417-63.

66. Sullivan R, Wilson DA, Feldon J, Yee BK, Meyer U, Richter-Levin G, Avi A, Michael T, Gruss M, Bock J, Helmeke C, Braun K. The International Society for Developmental Psychobiology annual meeting symposium: impact of early life experiences on brain and behavioral development. Dev Psychobiol. 2006;48:583-602.

67. Uriarte N, Breigeiron MK, Benetti F, Rosa XF, Lucion AB. Effects of maternal care on the development, emotionality, and reproductive functions in male and female rats. Dev Psychobiol. 2007;49:451-62.

68. Walker CD, Deschamps S, Proulx K, Tu M, Salzman C, Woodside B, Lupien S, Gallo-Payet N, Richard D. Mother to infant or infant to mother? Reciprocal regulation of responsiveness to stress in rodents and the implications for humans. J Psychiatry Neurosci. 2004;29: 364-82.

69. Webster JI, Sternberg EM. Role of the hypothalamic-pituitaryadrenal axis, glucocorticoids and glucocorticoid receptors in toxic sequelae of exposure to bacterial and viral products. J Endocrinol. 2004;181:207-21.

70. Weinstock M. Gender differences in the effects of prenatal stress on brain development and behaviour. Neurochem Res. 2007; 32:1730-40.

71. Zorrilla EP. Multiparous species present problems (and possibilities) to developmentalists. Dev Psychobiol. 1997;30:141-50.

72. Zuckerman L, Rehavi M, Nachman R, Weiner I. Immune activation during pregnancy in rats leads to a postpubertal emergence of disrupted latent inhibition, dopaminergic hyperfunction, and altered limbic morphology in the offspring: a novel neurodevelopmental model of schizophrenia. Neuropsychopharmacology. 2003;28:1778-89.

73. Zuckerman L, Weiner I. Post-pubertal emergence of disrupted latent inhibition following prenatal immune activation. Psychopharmacology 2003;169:308-13.

74. Zuckerman L, Weiner I. Maternal immune activation leads to behavioral and pharmacological changes in the adult offspring. J Psychiatr Res. 2005;39:311-23. 